EPJ Web of Conferences 81, 05020 (2014)

DOI: $10.1051 /$ epjconf/ 20148105020

(C) Owned by the authors, published by EDP Sciences, 2014

\title{
Interactions of light mesons with photons
}

\author{
Stefan Leupold ${ }^{1, a}$ \\ ${ }^{1}$ Department of Physics and Astronomy, Uppsala University, Sweden
}

\begin{abstract}
Interactions between hadrons and photons constitute an important tool to explore the intrinsic structure of hadrons. At the same time, high-precision determinations of electromagnetic processes, as in particular $g-2$ of the muon, constitute a promising tool to look for the impact of beyond-standard-model physics. Here hadronic contributions constitute the main uncertainty for the standard-model prediction. In this talk results for electromagnetic transition form factors and two-gamma amplitudes are presented.
\end{abstract}

\section{Motivation}

As is well-known by now hadrons are not elementary objects but have an intrinsic structure. To explore a complicated object it is certainly a good idea to use a probe that one understands much better than the object which one wants to study. This qualifies electromagnetism, i.e. real and virtual photons, as an excellent tool to study the structure of hadrons. Quantities of central importance are electromagnetic elastic and transition form factors of hadrons and two-photon amplitudes, the latter being related, e.g., to polarizabilities. Amusingly the logic to explore the less known hadrons by well-known electromagnetism gets another twist in the high-precision searches for low-energy traces of physics beyond the standard model. The required high-precision predictions of the standard model receive contributions from hadronic fluctuations. Examples for such an interplay can be observed for the rare pion decay into $e^{+} e^{-}$and for the gyromagnetic ratio of the muon; see, e.g., [1-3] and references therein. The key quantities are again the same: form factors and two-photon amplitudes.

In the last few years my collaborators and me have explored such quantities with two different tools: a Lagrangian and a dispersive approach. The results from the former approach are already well documented in the literature [4-11]. Therefore I will use the rest of these proceedings to discuss our recent work on the pion transition form factor based on dispersion theory [12].

\section{Pion transition form factor}

In his talk at this conference Peter Stoffer has explained in detail how the hadronic light-by-light contribution to $g-2$ of the muon involves the pion transition form factor; see Peter Stoffer's contribution to these proceedings and also [3]. For the standard-model prediction of the rare pion decay to $e^{+} e^{-}$the pion transition form factor constitutes the only non-trivial input at leading order in the electromagnetic interaction; see, e.g., $[13,14]$. From the hadronic point of view one interesting aspect is to figure out to which extent vector-meson dominance holds for the pion transition form factor $[2,7,9,11]$.

\footnotetext{
ae-mail: stefan.leupold@physics.uu.se
} 
The pion transition form factor is a function of the two photon virtualities, $F_{\pi}\left(q_{1}^{2}, q_{2}^{2}\right)$. Concerning experiments there are different kinematical regions where one can access the pion transition form factor. The reference/normalization point is provided by the pion decay into two real photons (or the corresponding formation process of photon fusion $2 \gamma \rightarrow \pi^{0}$ ). Due to the small pion mass the singlevirtual pion decay $\pi^{0} \rightarrow \gamma e^{+} e^{-}$and the double-virtual pion decay $\pi^{0} \rightarrow 2 e^{+} 2 e^{-}$have only little phase space. Better suited to explore the dependence on $q_{1}^{2}$ and $q_{2}^{2}$ are the production processes $e^{+} e^{-} \rightarrow \pi^{0} \gamma$ and $e^{+} e^{-} \rightarrow \pi^{0} e^{+} e^{-}$. If the initial leptons emit photons in flight that merge to a pion, one explores $F_{\pi}\left(q_{1}^{2}, q_{2}^{2}\right)$ for negative photon virtualities. Because of the amplitude suppressing photon propagators a significant contribution comes from quasi-real $q_{1}$ or $q_{2}$. Then one obtains information on $F_{\pi}\left(q^{2}, 0\right)$ with $q^{2}<0$. If the initial leptons annihilate into a virtual photon (with momentum $q_{1}$ ), which decays into a pion and a softer virtual or real photon (with momentum $q_{2}$ ), then one explores $F_{\pi}\left(q_{1}^{2}, q_{2}^{2}\right.$ ) for the time-like region $q_{1}^{2}>0$ and $q_{2}^{2} \geq 0$.

Theoretically the different kinematical regions will be approached step by step. At present I will concentrate on the time-like region, energies up to about $1 \mathrm{GeV}$ and a single-virtual form factor, i.e. on $F_{\pi}\left(q^{2}, 0\right)$ for $q^{2}>0$. Thus the corresponding experimental situation is $e^{+} e^{-} \rightarrow \pi^{0} \gamma$ and the total c.m.s. energy - which is just $\sqrt{q^{2}}-$ covers the masses of the vector mesons $\rho, \omega$ and $\phi$. The dispersive approach to the pion transition form factor [12] is based on preceding works of the Bonn group [15-17]. A similar approach for $\eta$ mesons instead of pions can be found in $[18,19]$.

The starting point for a dispersive approach is to find the relevant inelasticities for the process and kinematical region of interest. For the case at hand the virtual photon can be isoscalar or isovector. In the former/latter case it can couple to three/two pions. Up to $1 \mathrm{GeV}$ one can safely neglect four-pion states and kaons. (The $\pi^{0} \eta, 2 \pi^{0}$ and $3 \pi^{0}$ states are all forbidden by charge conjugation symmetry.) Thus for the isovector virtual photon one needs the following information: The amplitude for $e^{+} e^{-} \rightarrow$ $\pi^{+} \pi^{-}$and the amplitude for $\pi^{+} \pi^{-} \rightarrow \pi^{0} \gamma$. The latter has been addressed in [17] using dispersion theory to include all cross-channel rescattering of any pair of pions based on their $p$-wave interaction. The only input to this analysis is pion $p$-wave phase shift, which is very well known. Note that the $\rho$ meson is automatically included in this framework as the lowest resonance in the two-pion $p$-wave channel. The other required amplitude, $e^{+} e^{-} \rightarrow \pi^{+} \pi^{-}$, is nothing but the elastic pion vector form factor. It has already been used in the dispersive approach to the decays $\omega / \phi \rightarrow \pi^{0} \gamma^{*}$ [16]. Note that these decays are very similar to $e^{+} e^{-} \rightarrow \gamma^{*}(I=1) \rightarrow \pi^{0} \gamma$ because, assuming perfect isospin symmetry, the real photon has $I=0$ if the virtual one has isospin 1. Except for different kinematics and masses the real photon resembles the isoscalar vector mesons. Therefore the reaction amplitude for $e^{+} e^{-} \rightarrow \gamma^{*}(I=1) \rightarrow \pi^{0} \gamma$ can be determined in the very same way as the decay amplitudes for $\omega / \phi \rightarrow \pi^{0} \gamma^{*}$. To summarize, the contribution to the pion transition form factor $F_{\pi}\left(q^{2}, 0\right)$ is very well under control if the virtual photon is isovector and the dominant inelasticity is therefore $2 \pi$.

Unfortunately the situation is more complicated for the isoscalar case. The reason is that the relevant amplitudes are not of type two-body to two-body. Because of the three-pion intermediate state one has five- instead of four-point amplitudes. Scattering theory, partial-wave decomposition and dispersion theory are highly developed for four-point amplitudes (two-body to two-body reactions), but higher $n$-point functions are significantly more complicated. In principle, one needs the amplitudes for $e^{+} e^{-} \rightarrow \pi^{+} \pi^{-} \pi^{0}$ and $\pi^{+} \pi^{-} \pi^{0} \rightarrow \pi^{0} \gamma$. One might decompose the problem one step further: We need (a) the initial production of three pions from an isoscalar source, (b) the mutual interactions between the three pions (which are basically elastic in the considered energy range) and finally (c) the transition to the final state $\pi^{0} \gamma$. Part (b) can be addressed in a dispersive framework along the lines of [15]. Also part (c) fits nicely into this framework as the neutral pion of the three-pion intermediate state can be identified with the final-state pion. Any other option (charge transfer between the pions prior to the formation of the final state) is already covered by part (b). 
MESON 2014 $-13^{\text {th }}$ International Workshop on Production, Properties and Interaction of Mesons
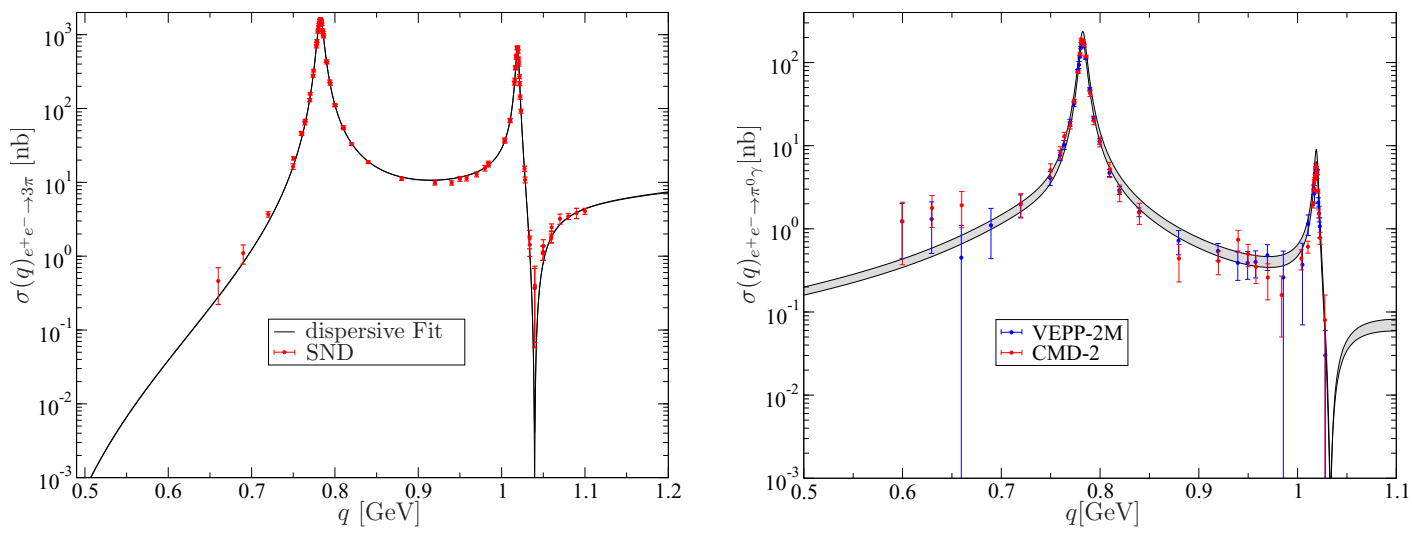

Figure 1. Left: Our fit to the cross section for $e^{+} e^{-} \rightarrow 3 \pi$ based on an analytically improved sum of Breit-Wigner functions for the narrow resonances $\omega$ and $\phi$ and a polynomial background. Data taken from [20,21]. Right: Our description of the cross section for $e^{+} e^{-} \rightarrow \pi^{0} \gamma$ without any further parameters. Data taken from [22-24].

The remaining problem is part (a), i.e. the question: How likely is it as a function of the photon energy to form a three-pion state? Phenomenology (see, e.g., the left-hand side of figure 1) tells us that this production process is dominated by the narrow vector mesons $\omega$ and $\phi$. Therefore we choose to parametrize the production amplitude by a sum of Breit-Wigner functions for the narrow resonances and a polynomial background. Once three pions are formed, their final-state interaction is treated by dispersion theory along the lines of [15]. The parameters are fitted to the cross section data for $e^{+} e^{-} \rightarrow \pi^{+} \pi^{-} \pi^{0}$. Actually we do not directly use the Breit-Wigner functions but feed their imaginary part into a dispersion relation to ensure reasonable analytic properties of our input. (The pure inclusion of a width with the correct phase-space behavior in a propagator without the corresponding real part of the self energy violates analyticity [25].) Furthermore, the polynomial background is constrained by the axial anomaly, which predicts the size of the amplitude $\gamma^{*}-3 \pi$ at low energies $[11,17]$. We limit the polynomial to a linear function. In case of an insufficient fit quality one can use a higher-order polynomial. Our parametrization has then the following three fit parameters: The respective coupling strengths of $\omega$ and $\phi$ and the coefficient of the linear term of the polynomial. The masses and relevant partial decay widths of the resonances are not fitted but taken from the Particle Data Group [26]. The energy dependence of the three-pion decays is taken from the dispersive analysis [15]. The quality of the fit is displayed in figure 1.

With a parametrization for the amplitude $e^{+} e^{-} \rightarrow \pi^{+} \pi^{-} \pi^{0}$ and otherwise a fully self-contained dispersive treatment of $\pi^{+} \pi^{-} \pi^{0} \rightarrow \pi^{0} \gamma$ and $e^{+} e^{-} \rightarrow \pi^{+} \pi^{-} \rightarrow \pi^{0} \gamma$ we have all ingredients for a calculation of the single-virtual pion transition form factor at low energies. It is the only non-trivial quantity that enters the cross section for the reaction $e^{+} e^{-} \rightarrow \pi^{0} \gamma$. The result for this cross section is compared to data on the right-hand side of figure 1 . Obviously one obtains a very satisfying description of the data without any parameters that were directly fitted to these data. The gray uncertainty band of $10 \%$ is motivated by the finding in [16] that an unsubtracted dispersion relation reproduces the decay widths $\omega / \phi \rightarrow \pi^{0} \gamma$ within $10 \%$. A more thorough error analysis will be presented in [12].

The next steps are extensions to space-like momenta and double virtuality of the photons. Another room for improvement lies in the fact that with the present parametrization the transition form factor does not show a decent high-energy behavior. This is not a principle problem for a low-energy calculation. Using only two- and three-pion intermediate states limits the approach to low energies 
anyway. Nonetheless a smooth matching to the high-energy limit dictated by QCD would be more appreciable. A possible future project utilizing the same machinery could be a prediction for the Dalitz plot distribution $\omega \rightarrow \gamma \pi \pi$. This process is in turn related to $\omega \rightarrow 3 \gamma$ from a dispersive point of view, which connects back to virtual light-by-light scattering and therefore to $g-2$ of the muon.

\section{Acknowledgements}

I would like to thank the organizers for their kind invitation and the excellent conference. The results that I had the pleasure to present at the MESON 2014 would not have been possible without my collaborators I. Danilkin, F. Eichstädt, P. Engström, H. Ghaderi, M.F.M. Lutz, M. Hoferichter, B. Kubis, F. Niecknig, S.P. Schneider, B. Strandberg and C. Terschlüsen.

\section{References}

[1] F. Jegerlehner, A. Nyffeler, Phys.Rept. 477, 1 (2009), 0902 . 3360

[2] E. Czerwinski et al. (2012), 1207.6556

[3] G. Colangelo, M. Hoferichter, B. Kubis, M. Procura, P. Stoffer (2014), 1408. 2517

[4] M.F.M. Lutz, S. Leupold, Nucl.Phys. A813, 96 (2008), 0801.3821

[5] S. Leupold, M.F.M. Lutz, Eur.Phys.J. A39, 205 (2009), 0807 . 4686

[6] S. Leupold, Phys.Rev. D80, 114012 (2009), 0907.0100

[7] C. Terschlüsen, S. Leupold, Phys.Lett. B691, 191 (2010), 1003.1030

[8] C. Terschlüsen, S. Leupold, M.F.M. Lutz, Eur.Phys.J. A48, 190 (2012), 1204.4125

[9] S. Leupold, C. Terschlüsen, PoS BORMIO2012, 024 (2012), 1206. 2253

[10] I. Danilkin, M.F.M. Lutz, S. Leupold, C. Terschlüsen, Eur.Phys.J. C73, 2358 (2013), 1211.1503

[11] C. Terschlüsen, B. Strandberg, S. Leupold, F. Eichstädt, Eur.Phys.J. A49, 116 (2013), 1305.1181

[12] M. Hoferichter, B. Kubis, S. Leupold, F. Niecknig, S.P. Schneider, in preparation (2014)

[13] P. Vasko, J. Novotny, JHEP 1110, 122 (2011), 1106. 5956

[14] T. Husek, K. Kampf, J. Novotny (2014), 1405.6927

[15] F. Niecknig, B. Kubis, S.P. Schneider, Eur.Phys.J. C72, 2014 (2012), 1203.2501

[16] S.P. Schneider, B. Kubis, F. Niecknig, Phys.Rev. D86, 054013 (2012), 1206. 3098

[17] M. Hoferichter, B. Kubis, D. Sakkas, Phys.Rev. D86, 116009 (2012), 1210.6793

[18] F. Stollenwerk, C. Hanhart, A. Kupsc, U.G. Meißner, A. Wirzba, Phys.Lett. B707, 184 (2012), 1108.2419

[19] C. Hanhart, A. Kupsc, U.G. Meißner, F. Stollenwerk, A. Wirzba, Eur.Phys.J. C73, 2668 (2013), 1307.5654

[20] M. Achasov, V. Aulchenko, K. Beloborodov, A. Berdyugin, A. Bogdanchikov et al., Phys.Rev. D66, 032001 (2002), hep-ex/0201040

[21] M. Achasov, K. Beloborodov, A. Berdyugin, A. Bogdanchikov, A. Bozhenok et al., Phys.Rev. D68, 052006 (2003), hep-ex/0305049

[22] M. Achasov, A. Berdyugin, A. Bozhenok, D. Bukin, S. Burdin et al., Eur.Phys.J. C12, 25 (2000)

[23] M. Achasov, K. Beloborodov, A. Berdugin, A. Bogdanchikov, A. Bozhenok et al., Phys.Lett. B559, 171 (2003), hep-ex/0302004

[24] R. Akhmetshin et al. (CMD2 Collaboration), Phys.Lett. B605, 26 (2005), hep-ex/0409030

[25] P.A. Henning, Nucl.Phys. A546, 653 (1992)

[26] K. Olive et al. (Particle Data Group), Chin.Phys. C38, 090001 (2014) 